\title{
Rewiev
}

\section{Mutations in type I collagen genes resulting in osteogenesis imperfecta in humans ${ }^{\$}$}

\author{
Anna Gajko-Galicka ${ }^{\bowtie}$
}

Department of Medical Chemistry, Medical Academy of Biatystok, 15-230 Biatystok 8, Poland

Received: 7 February, 2002; revised: 15 February, 2002; accepted: 22 April, 2002

Key words: type I collagen, mutation, osteogenesis imperfecta

\begin{abstract}
Osteogenesis imperfecta (OI), commonly known as "brittle bone disease", is a dominant autosomal disorder characterized by bone fragility and abnormalities of connective tissue. Biochemical and molecular genetic studies have shown that the vast majority of affected individuals have mutations in either the COL1A1 or COL1A2 genes that encode the chains of type I procollagen. OI is associated with a wide spectrum of phenotypes varying from mild to severe and lethal conditions. The mild forms are usually caused by mutations which inactivate one allele of $C O L 1 A 1$ gene and result in a reduced amount of normal type I collagen, while the severe and lethal forms result from dominant negative mutations in $C O L 1 A 1$ or $C O L 1 A 2$ which produce structural defects in the collagen molecule. The most common mutations are substitutions of glycine residues, which are crucial to formation and function of the collagen triple helix, by larger amino acids. Although type I collagen is the major structural protein of both bone and skin, the mutations in type I collagen genes cause a bone disease. Some reports showed that the mutant collagen can be expressed differently in bone and in skin. Since most mutations identified in OI are dominant negative, the gene therapy requires a fundamentally different approach from that used for genetic-recessive disorders. The antisense therapy, by reducing the expression of mutant genes, is able to change a structural mutation into a null mutation, and thus convert severe forms of the disease into mild OI type I.
\end{abstract}

\footnotetext{
${ }^{\$}$ This work was supported by a project (No. 3-03-405) from Medical Academy in Bialystok, Poland.

${ }^{\otimes}$ Anna Gajko-Galicka, Department of Medical Chemistry, Medical Academy of Białystok, 15-230 Białystok 8, Poland, tel.: (48 85) 742 2059, fax: (48 85) 742 4907, e-mail: angajko@amb.ac.bialystok.pl Abbreviation: OI, osteogenesis imperfecta.
} 


\section{OSTEOGENESIS IMPERFECTA - "BRITTLE BONE DISEASE"}

Osteogenesis imperfecta (OI) is a heterogeneous genetic disorder of connective tissue, commonly known as "brittle bone disease". The major clinical feature that defines OI is bone fragility. Fractures may be rare or frequent (more than 200 prior to puberty) and bone fragility may be accompanied with reduced life span and various connective tissue abnormalities. On the basis of clinical, radiologic and genetic criteria, Sillence et al. (1979) identified four types of OI (Table 1). The mildest form is OI type I characterized by blue sclerae, premature deafness, and mild to moderate bone fragility. It is subclassified as IA if the teeth are normal, or IB if there is there is usually moderate bowing of long bones, and the fracture rate decreases around puberty. Many affected individuals have moderately short stature, osteoporosis and mild scoliosis. Recently, two groups of patients initially classified as OI type IV, turned out to have unique clinical and histological features. These new forms have subsequently been named types V (Glorieux et al., 2000) and VI (Ward et al., 2000). OI affects probably more than one per 10000 individuals; some persons, however, are not diagnosed until later on in their lives.

Biochemical and molecular genetic studies have shown that the vast majority of individuals (>90\%) affected with OI types I-IV, have mutations in either the COL $1 A 1$ or COL $1 A 2$ genes that encode the chains of type I pro-

Table 1. Classification of OI phenotype according to Sillence et al. (1979)

\begin{tabular}{|c|c|c|}
\hline OI type & Inheritance & Clinical characteristics \\
\hline I & $\mathrm{AD}$ & Normal stature, little or no deformity, blue sclerae, hearing loss \\
\hline II & $\mathrm{AD}$ (new mutations) & Lethal in perinatal period, beaded ribs, long bone fractures \\
\hline III & $\begin{array}{l}\mathrm{AD} \\
\mathrm{AR} \text { (rare) }\end{array}$ & $\begin{array}{l}\text { Progressively deforming, short stature, multilpe fractures, triangular fa- } \\
\text { cies, hearing loss }\end{array}$ \\
\hline IV & $\mathrm{AD}$ & $\begin{array}{l}\text { Moderately severe, variable short stature, dentinogenesis imperfecta, os- } \\
\text { teoporosis, bowing of long bones }\end{array}$ \\
\hline
\end{tabular}

$\mathrm{AD}$, autosomal dominant; $\mathrm{AR}$, autosomal recessive.

dentinogenesis imperfecta. The most severe form is the lethal perinatal form, OI II. Infants with type II OI experience intrauterine fractures, intracranial hemorrhage after vaginal delivery, and succumb to death shortly after birth. The children with type III OI may die in infancy of respiratory problems, and many others will die in their childhood from pneumonia, cor pulmonale, or trauma such as skull fracture. Those infants who survive suffer from gradual deformity of the long bones and spine due to fractures; they require multiple orthopedic rodding procedures and wheelchairs for mobility. Type IV OI is the least common and has some similarities to mild type I and type III phenotypes. In childhood, collagen, the major structural protein of bone, skin and tendons. The genetic defect underlying OI types V and VI remains to be elucidated, as it does not appear to be associated with collagen type I mutations. In many cases, OI is inherited in an autosomal dominant manner but new mutations are frequent. A few cases result from autosomal recessive inheritance. Some patients suffer from OI as a result of mosaicism in a parent who shows little or no clinical abnormality (Zlotogora, 1998). Since so far osteogenesis imperfecta has been an incurable genetic disease, cell therapy and gene therapy are being investigated as potential treatments. 


\section{THE TYPES OF MUTATIONS IDENTIFIED IN OSTEOGENESIS IMPERFECTA}

Mutations in type I collagen genes resulting in OI can be considered in two major categories, mutations that resulted in exclusion of the product of the mutant allele from the mature collagen molecule, and those which permitted the incorporation of a structurally abnormal chain, referred to by Sykes (1985) as "excluded" and "included" mutations, respectively. In the first group, lack of expression of mutant product usually results from heterozygosity for premature termination codons in the COL1A1 gene (Willing et al., 1996). The "included mutations" result in the generation of abnormal type I procollagen molecules. The triple helical portion of either collagen chain contains 338 uninterrupted repeats of the triplet GXY, where $\mathrm{G}$ is glycine, $\mathrm{X}$ is often proline, and $\mathrm{Y}$ is often hydroxyproline. The presence of glycine, which has the smallest side chain in every third residue, is a prerequisite for correct folding of the three $\alpha$ chains into a collagen triple helix. The great majority of mutations appear to result in substitutions for glycine residues within the triple helix or if mutations occur in the consensus splice donor or acceptor sites, are the product of exon skipping events (Cole, 1994; Dalgleish, 1998). Multiexon rearrangements, shorter deletions, insertions or frameshift mutations are rare.

\section{EFFECT OF MUTATIONS ON THE EXPRESSION OF TYPE I COLLAGEN}

A description of type I collagen genes and the manner in which the collagen is synthesized and processed by the cell might help in understanding how mutations in these genes result in OI. Two genes, COL1A1 located on chromosome 17, and COL1A2 located on chromosome 7 , encode the pro $\alpha 1$ (I) and pro $\alpha 2(\mathrm{I})$ chains of type I procollagen, respec- tively (Kielty et al., 1993). These genes are composed of about 50 exons scattered over 18 $\mathrm{kb}$ (for $\alpha 1(\mathrm{I})$ ) and $38 \mathrm{~kb}$ (for $\alpha 2(\mathrm{I})$ ) of chromosomal material. Most of the exons consist of 54 or 108 base pairs, and the final coding mRNAs in the cytoplasm range in size from 5.5 to $7.2 \mathrm{~kb}$. Fibril-forming collagens such as type I collagen are synthesized into larger precursors, known as procollagens, which contain globular N-terminal and C-terminal propeptides. Two pro $\alpha 1$ and a single pro $\alpha 2$ chains first associate by hydrophobic and electrostatic interactions among the C-propeptides (Fig. 1A). The association is then stabilized by the formation of interchain disulphide bonds. From the carboxyl end, helix formation proceeds towards the amino terminal end in a zipper-like fashion. Simultaneously, proline and lysine residues are hydroxylated by specific hydroxylases and hydroxylysine residues are glycosylated by sugar transferases. These modifications occur only in chains that are in nonhelical configuration and cease as soon as the protein folds into a triple helix. In the extracellular space, specific $\mathrm{N}$ - and C-peptidases cleave the terminal extensions. The mature helical molecules self-aggregate into highly ordered fibrils stabilized by intermolecular cross-links formed by oxidative deamination of lysine and hydroxylysine residues. Mechanical strength of connective tissue is due mainly to fibrils which form a template for matrix deposition. In bone, the fibrils are the template for mineralization, i.e., in this case, for incorporation of hydroxyapatite crystals. The mechanical properties of bone are dependent on an intimate association between the collagen fibrils and crystals of hydroxyapatite.

The "excluded mutations" usually result in one null COL1A1 allele and, although only half of the normal amount of type I procollagen becomes synthesized, its structure is normal. The remaining mutations, which alter the primary structure of collagen, affect the protein in several ways. Almost all molecules that contain chains with mutations 


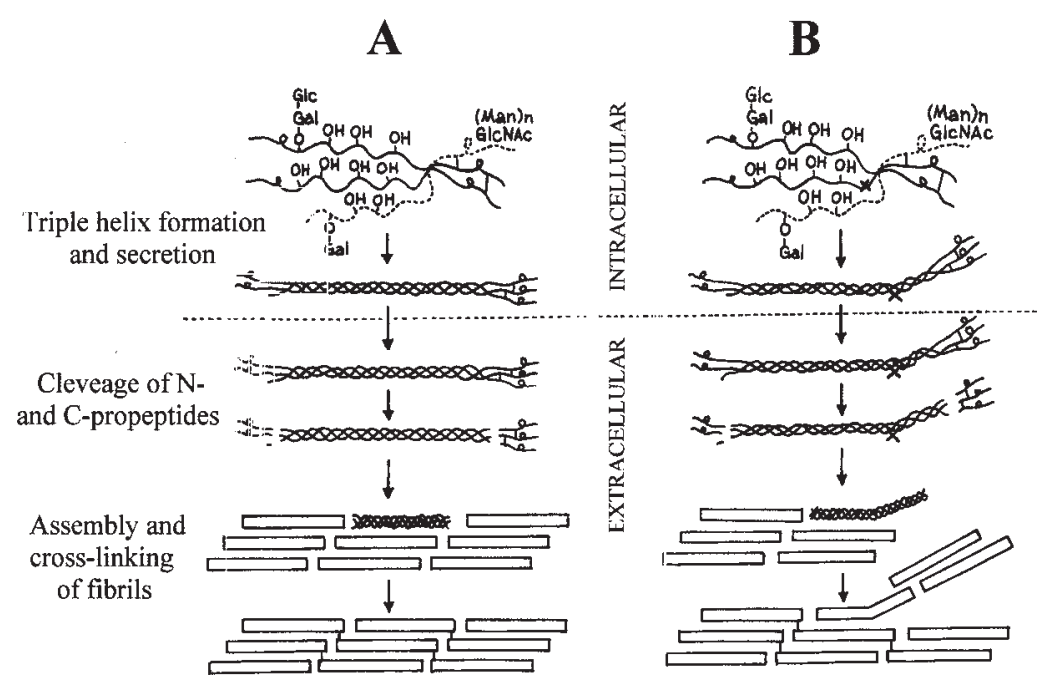

Figure 1A. Schematic representation of the intracellular and extracellular steps involved in the synthesis, processing, and assembly of type I collagen molecules into fibrils. B. Mutation which produces a conformational change such as a kink leading to the defective fibril formation.

The sign $(\times)$ in the procollagen chain indicates the point mutation.

in the triple-helical domain, are less stable than their normal counterparts (Baker et al., 1989). Another consistent observation is the overmodification of the portions of the $\alpha$-chains $\mathrm{N}$-terminal to the mutation (Lehmann et al., 1995) as judged by the lower electrophoretic mobility of the collagen chains on SDS/PAGE (Fig. 2). Since post-translational modification of pro $\alpha$ chains occurs only in the unfolded state, the unidirectional overmodification of lysyl and hydroxylysyl residues $\mathrm{N}$-terminal to the point mutation (as opposed to the normal modification C-terminal to the mutated site) has been interpreted as a result of delayed folding $\mathrm{N}$-terminal from the site of the mutations (Raughunath et al., 1994). The mutations which occur within the domains that encode the carboxyl-terminal propeptide may alter the ability of chains to aggregate into molecules (Chessler et al., 1993). Some substitutions for obligate glycines interrupt the zipper-like folding of the triple helix and generate unfolded procollagen that first accumulates in fibroblasts and then is degraded. The effects of the mutations are amplified because both the normal and mutated chains present in the same molecule are degraded in a process re-
$\mathbf{A}$

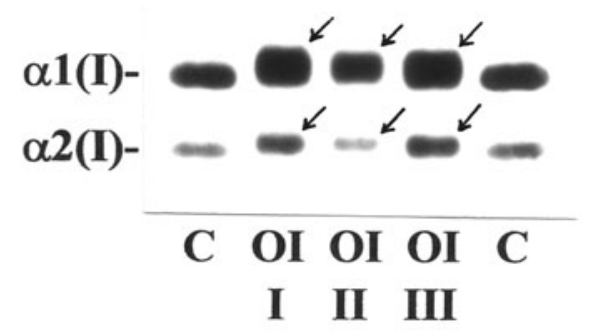

B

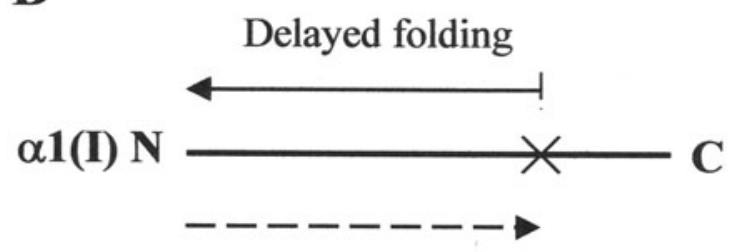

Gradient of overmodification

Figure 2A. SDS/PAGE of type I collagen secreted by fibroblasts from three infants with lethal type of disease (OI I, OI II, and OI III, respectively) and a control (C).

Cells were incubated with $\left[{ }^{3} \mathrm{H}\right]$ proline overnight in the presence of ascorbic acid. Arrows indicate the overmodified forms of the $\alpha 1$ (I) and $\alpha 2$ (I) chains present in the OI media.

B. Helix formation from carboxyl (C) to amino (N) ends (delayed from the point mutation) and gradient of overmodification (in opposite direction) due to the point mutation $(X)$ in the collagen chain. 
ferred to as "procollagen suicide" or a dominant negative effect (Prockop \& Kivirikko, 1984). Other consequences of mutations include defective processing of extracellular type I procollagen by N-proteinase (Vogel et al., 1988) or increased susceptibility of trimers to proteases (Valli et al., 1993). Studies on fibril formation demonstrated that molecules with such a mutation that introduced a flexible kink into the triple helix, copolymerized with the normal molecules (Fig. 1B). The presence of the kinked mole-

\section{TRANSLATION OF MUTATION TO PHENOTYPE}

The phenotypic consequences of mutations in type I collagen genes reflect the type of mutation, gene in which the mutation occurred and location of the mutation. Mutations in the COL1A1 gene resulting in the synthesis of half the normal amount of functional pro $\alpha 1$ (I) chains (because of one null allele) have but mild clinical consequences found in individuals with type I OI (Fig. 3). Normal type I
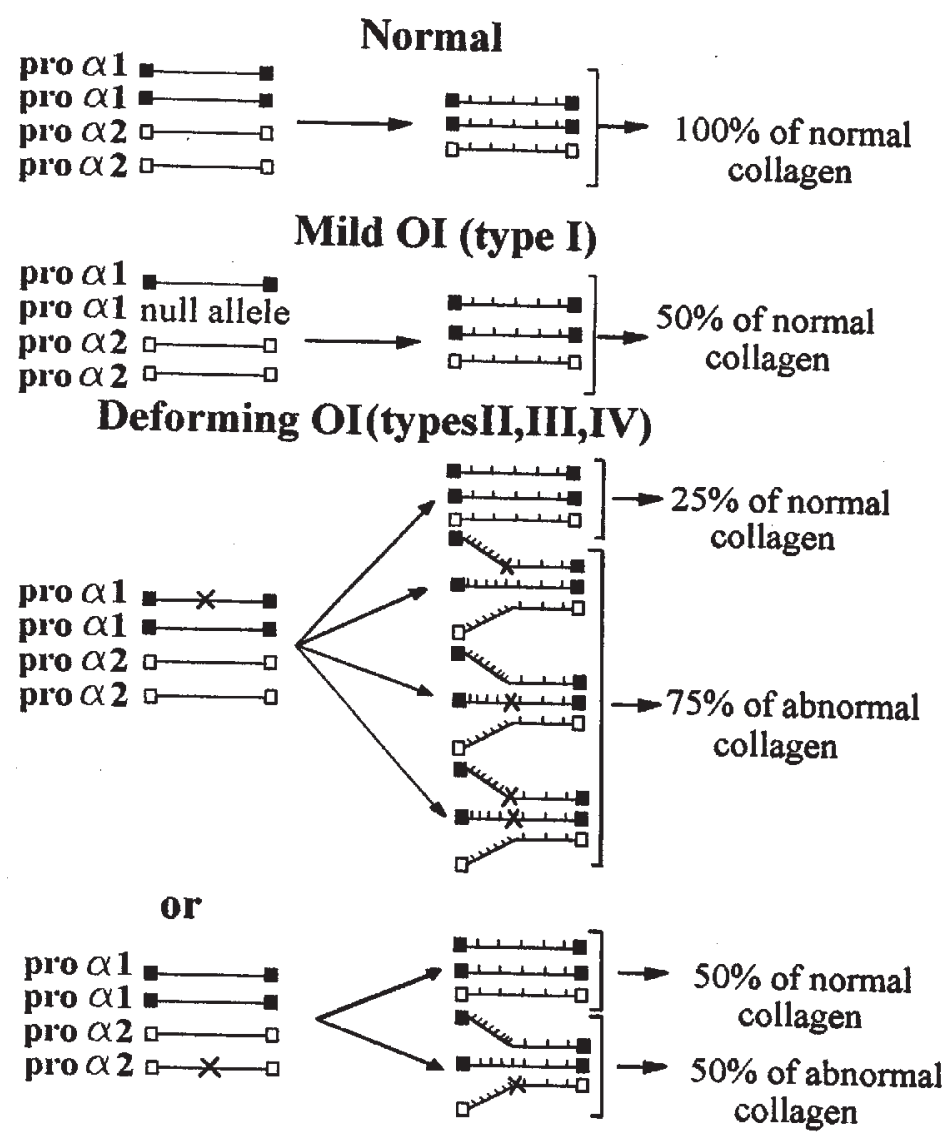

Figure 3. The molecular mechanism of OI.

Mutations which decrease the synthesis of normal type I collagen to $50 \%$ (because of one null allele of COL1A1 gene) result in mild type I OI. The mutations that affect the structure of the procollagen chains have more deleterious phenotypic consequences (types II, III and IV OI). The gene, in which the mutation occurs, affects the proportion of abnormal molecules and is probably reflected in the phenotype. The signs $(x)$ indicate mutations in the procollagen chains; the small vertical lines indicate the posttranslational modifications, increasing from the amino end to the point mutation.

cules delayed fibril formation, reduced the total amount of collagen incorporated into the fibrils, and drastically altered the morphology of the fibrils (Vogel et al., 1988). Since the integrity, architecture and functions of connective tissues are the result of specific interactions between collagen, proteoglycans and structural glycoproteins, the presence of abnormal collagen chains may have a strong influence on the metabolism of noncollagenous components (Tenni et al., 1988). procollagen molecules require a minimum of two pro $\alpha 1$ (I) chains for stability and when the number of chains available is halved, the amount of type I procollagen produced is diminished to $50 \%$ of normal. The phenotypic effects of "included mutations" that result in the generation of abnormal type I procollagen molecules are more deleterious than those of null mutations. If an abnormal chain leads to very rapid intracellular degradation of molecules that incorporate the chain, the clinical 
consequences would differ, depending on the gene in which the mutation did occur. Mutations in the COL1A1 gene may be highly deleterious, and even lethal, because they comprise three-quarters of all the procollagen molecules of type I synthesized (Fig. 3). In contrast, similar mutations in the COL1A2 gene would result in the loss of only half the molecules made and so might be similar in effect to a null COL1A1 allele.

It has also been proposed that the position of a mutation along the chains may be a crucial factor, with mutations closer to the carboxyl end resulting in a more severe phenotype than mutations near the amino terminal end of the chain (Starman et al., 1989). This gradient is modified by the nature of the substituting amino acid, so that some of them may be lethal when incorporated at any place along the entire domain (e.g., aspartic acid) while others may show a lethal to a nonlethal transition in the carboxyl terminal half of the chain (e.g., cysteine). Such gradients are not apparent in the case of other substitutions although domains producing lethal and nonlethal phenotypes have been proposed to exist in the $\alpha 2(\mathrm{I})$ chain (Marini et al., 1993). Functions associated with crucial versus noncrucial domains might include their role in thermal stability of collagen, its secretion from the cell or interactions with collagenous or noncollagenous molecules in the extracellular matrix. The genetic background and other modifiers appear to be important, as the same mutation has been observed to result in different phenotypes (Zhuang et al., 1996). We have found that defects in collagen metabolism (produced by substitution of arginine for glycine at position 388 in the $\alpha 1$ (I) chain (unpublished) are correlated with a decrease in the activity of prolidase, an enzyme essential for collagen synthesis and cell growth. This observation might point to another an important mechanism being involved in producing the OI phenotype (Galicka et al., 2001).

\section{WHY MUTATIONS IN TYPE I COLLAGEN GENES CAUSE BONE DISEASE?}

The type I procollagen is the major structural protein of skin, bone and tendons. So far, most studies have been focused on procollagen synthesized by fibroblasts cultured from skin biopsies of OI patients. Genomic mutations are presumably expressed in all type I collagen-producing tissues, but OI primarily affects the skeletal system. Therefore, to study the mechanism through which collagen mutations lead to the clinical phenotype of OI, the most appropriate cells are osteoblasts, as they are of greatest significance for skeletal pathophysiology. Some reports suggested that the patient's osteoblasts expressed type I collagen in a different way than did fibroblasts. In patients with type IV OI, the osteoblasts contained a higher proportion of mutant collagen as compared to the fibroblasts (Sarafova et al., 1998; Galicka et al., 2002). We found that the mutant collagen was secreted more rapidly by bone than by skin cells (Galicka et al., 2002). Furthermore, the osteoblast mutant collagen molecules showed higher thermal stability (Sarafova et al., 1998; Galicka et al., 2002). The presence of mutant collagen in bone, but not in skin matrix, suggests that the incorporation of abnormal molecules is tissue specific (Mundlos et al., 1996). It has been shown that mutant molecules are incorporated into the extracellular matrix of bone efficiently and represent a high percentage of total collagen isolated from whole cortical bone (Niyibizi et al., 1992). The copolymerization of normal and mutated molecules which induced formation of poor and disorganized matrix, had a dramatic effect on mineral deposition (Cohen-Solal et al., 1994).

While type I collagen is the major organic component of both skin and bone, there are many differences between these tissues in the composition of other matrix components. The 
noncollagenous components of bone, involved in matrix organization and mineralization, include: osteonectin, which acts as a nucleator in collagen mediated mineralization, decorin, which is important in fibril formation, the large chondroitin sulfate proteoglycan and hyaluronan, which can "capture space" for subsequent matrix deposition, as well as biglycan associated with osteoblast differentiation (Scott, 1988). Studies on bone matrix of OI patients showed not only reduced levels of some components (collagen, osteonectin, the large chondroitin sulfate proteoglycan, biglycan, and decorin) in bone cells, but also elevation of some other matrix components (thrombospondin, fibronectin, and hyaluronan) (Fedarko et al., 1995). These quantitative changes of matrix components in OI bone, in addition to the mutant collagen, may play a significant role in OI bone pathology. Finally, the bone requirements for molecular structure may be greater than those of skin and other soft tissues, and this might explain, in part, the relative tissue specificity of the mutations discussed.

\section{APPROACHES TO THE GENE THERAPY OF OSTEOGENESIS IMPERFECTA}

The experiments with expression of collagen genes with mutations in transgenic mice directly confirmed the conclusion that the mutations in the genes for type I procollagen lead to the OI phenotype (Forlino et al., 1999). Transgenic mice are particularly useful for both studying the consequences of disease-causing mutations in matrix genes and development of approaches to the gene therapy of the disorder. Since most mutations in OI are dominant negative, it is difficult to target them by gene therapy because supplying the normal gene without silencing the abnormal gene is ineffective. By elimination or reduction of the expression of mutant genes, gene therapy is able to create a functionally null allele and convert the clinically severe forms of OI into the biochemical equivalent of mild OI type I. One approach to mutation suppression involves the use of antisense oligonucleotides which bind to the target mRNA for the mutant protein and prevent its translation. Because of the highly repetitive nature of the type I collagen genes, the specificity of mutant allele suppression observed in the antisense oligonucleotide experiments appears to be insufficient for therapeutic trials (Wang \& Marini, 1996). To increase specificity, an alternative approach of antisense therapy has been investigated using ribozymes. Selective cleveage of mutant type I collagen RNAs in cell-free assays (Grassi et al., 1997) and in cultured OI fibroblasts (Dawson \& Marini, 2000) was achieved using hammerhead ribozymes. Thus ribozymes seem to be promising future agents for the gene therapy of dominant negative genetic disorders, such as osteogenesis imperfecta.

\section{R E F E R E N C E S}

Baker AT, Ramshaw JAM, Chan D, Cole WG, Bateman JF. (1989) Changes in collagen stability and folding in lethal perinatal osteogenesis imperfecta. The effect of $\alpha 1$ (I)-chain glycine-to-arginine substitutions. Biochem J.; 261: 253-7.

Chessler SD, Wallis GA, Byers PH. (1993) Mutations in the carboxyl-terminal propeptide of the pro $\alpha 1$ (I) chain of type I collagen result in defective chain association and produce lethal osteogenesis imperfecta. J Biol Chem.; 268: 18218-25.

Cohen-Solal L, Zylberberg L, Sangalli A, Gomez Lira M, Mottes M. (1994) Substitution of aspartic acid for glycine 700 in the $\alpha 2$ (I) chain of type I collagen in a recurrent lethal type II osteogenesis imperfecta dramatically affects the mineralization of bone. J Biol Chem.; 269: $14751-8$.

Cole WG. (1994) Collagen genes: mutations affecting collagen structure and expression. Prog Nucl Acid Res Mol Biol.; 47: 29-80. 
Dalgleish R. (1998) The human type I collagen mutation database. Nucleic Acids Res.; 26: 253-5.

Dawson P, Marini JC. (2000) Hammerhead ribozymes selectively suppress mutant type I collagen mRNA in osteogenesis imperfecta fibroblasts. Nucleic Acids Res.; 28: 4013-20.

Fedarko NS, Robey PG, Vetter UK. (1995) Extracellular matrix stechiometry in osteoblasts from patients with osteogenesis imperfecta. J Bone Miner Res.; 10: 1122-9.

Forlino A, Porter FD, Lee EJ, Westphal H, Marini JC. (1999) Use of the Cre/lox recombination system to develop a non-lethal knock-in murine model for osteogenesis imperfecta with an $\alpha 1$ (I) G349C substitution. J Biol Chem.; 274: 37923-31.

Galicka A, Wolczynski S, Gindzienski A. (2002) Comparative studies of osteoblast and fibroblast type I collagen in a patient with osteogenesis imperfecta type IV. J Pathol.; 196: $235-7$.

Galicka A, Wolczynski S, Anchim T, Surazynski A, Lesniewicz R, Palka J. (2001) Defects of type I procollagen metabolism correlated with decrease of prolidase activity in a case of lethal osteogenesis imperfecta. Eur $J$ Biochem.; 268: 2172-8.

Glorieux FH, Rauch F, Plotkin H, Ward L, Travers R, Roughley PJ, Lalic L, Glorieux DF, Fassier F, Bishop N. (2000) Osteogenesis imperfecta type V: a new form of brittle bone disease. J Bone Min Res.; 15: 1650-8.

Grassi G, Forlino A, Marini JC. (1997) Cleavage of collagen RNA transcripts by hammerhead ribozymes in vitro is mutation-specific and shows competitive binding effects. Nucleic Acids Res.; 25: 3451-38.

Kielty CM, Hopkinson I, Grant ME. (1993) Collagen: the collagen family: structure, assembly, and organization in the extracellular matrix. In Connective tissue and its heritable disorders: Molecular, genetic and medical aspects. Royce PM, Steinmann B. eds, pp 103-47, Wiley-Liss New York.

Lehmann HW, Rimek D, Bodo M, Brenner RE, Vetter U, Worsdorfer O, Karbowski A, Muller
PK. (1995) Hydroxylation of collagen type I: evidence that both lysyl and prolyl residues are overhydroxylated in osteogenesis imperfecta. Eur J Clin Invest.; 25: 306-10.

Marini JC, Lewis MB, Wang Q, Chen KJ, Orrison BM. (1993) Serine for glycine substitution in type I collagen in two cases of type IV osteogenesis imperfecta (OI): additional evidence for a regional model of OI pathophysiology. J Biol Chem.; 268: 2667-73.

Mundlos S, Chan D, Weng YM, Sillence DO, Cole WG, Bateman JF. (1996) Multiexon deletions in the type I collagen COL1A2 gene in osteogenesis imperfecta type IB. Molecules containing the shortened $\alpha 2(\mathrm{I})$ chains show differential incorporation into the bone and skin extracellular matrix. J Biol Chem.; 271: 21068-74.

Niyibizi Ch, Bonadio J, Byers PH, Eyre DR. (1992) Incorporation of type I collagen molecules that contain a mutant $\alpha 2$ (I) chain (Gly $580 \rightarrow$ Asp) into bone matrix in a lethal case of osteogenesis imperfecta. $J$ Biol Chem.; 267: 23108-12.

Prockop DJ, Kivirikko KL. (1984) Heritable diseases of collagen. $N$ Engl $J$ Med.; 311: 376-86.

Raughunath M, Bruckner P, Steinmann B. (1994) Delayed triple helix formation of mutant collagen from patients with osteogenesis imperfecta. J Mol Biol.; 236: 940-9.

Sarafova AP, Choi H, Forlino A, Gajko A, Cabral WA, Tosi L, Reing CM, Marini JC. (1998) Three novel type I collagen mutations in osteogenesis imperfecta type IV probands are associated with discrepancies between electrophoretic migration of osteoblast and fibroblast collagen. Hum Mut.; 11: 395-403.

Scott JE. (1988) Proteoglycan-fibrillar collagen interactions. Biochem J.; 252: 313-23.

Sillence DO, Senn A, Danks DM. (1979) Genetic heterogeneity in osteogenesis imperfecta. J Med Genet.; 16: 101-16.

Starman BJ, Eyre D, Charbonneau H, Harrylock M, Weis MA, Weiss L, Graham JM. Byers PH. (1989) The position of substi- 
tution for glycine by cysteine in the triple helical domain of the pro $\alpha 1$ (I) chains of type I collagen determines the clinical phenotype. J Clin Invest.; 84: 1206-14.

Sykes B. (1985) The molecular genetics of collagen. BioEssays; 3: 112-7.

Tenni R, Cetta G, Dyne K, Rossi A, Quacci D, Lenzi L, Castellani AA. (1988) Type I procollagen in the severe non-lethal form of osteogenesis imperfecta. Defective pro- $\alpha 1$ (I) chains in a patient with abnormal proteoglycan metabolism and mineral deposits in the dermis. Hum Genet.; 79: 245-50.

Valli M, Zolezzi F, Mottes M, Antoniazzi F, Stanzial F, Tenni R, Pignatti P, Cetta G. (1993) Gly85 to Val substitution in pro $\alpha 1(\mathrm{I})$ chain causes mild osteogenesis imperfecta and introduces a susceptibility to protease digestion. Eur J Biochem.; 217: 77-82.

Vogel BE, Doelz R, Kadler KE, Hojima Y, Engel J, Prockop DJ. (1988) A substitution of cysteine for glycine 748 of the $\alpha 1$ chain produces a kink at this site in the procollagen molecule and an altered N-proteinase cleveage site over $225 \mathrm{~nm}$ away. $J$ Biol Chem.; 263: 19249-55.
Wang Q, Marini JC. (1996) Antisense oligodeoxynucleotides selectively suppress expression of the mutant $\alpha 2(\mathrm{I})$ collagen allele in type IV osteogenesis imperfecta fibroblasts. A molecular approach to therapeutics of dominant negative disorders. J Clin Invest.; 97: 448-54.

Ward L, Rauch F, Travers R, Lalic L, Alos N, Lalic R, Roughley PJ, Glorieux FH. (2000) Osteogenesis imperfecta type VI: a form of brittle bone disease with a mineralization defect. J Bone Min Res.; 15: M116.

Willing MC, Deschenes SP, Slayton RL, Roberts EJ. (1996) Premature chain termination is a unifying mechanism for COL1A1 null alleles in osteogenesis imperfecta type I cell strains. Am J Hum Genet.; 59: 799-809.

Zhuang J, Tromp G, Kuivaniemi H, Castells S, Prockop DJ. (1996) Substitution of arginine for glycine at position 154 of the $\alpha 1$ chain of type I collagen in a variant of osteogenesis imperfecta: comparison to previous cases with the same mutation. Am J Med Genet.; 61: 111-6.

Zlotogora J. (1998) Germ line mosaicism. Hum Genet.; 102: 381-6. 